\title{
The double scaling limit method in the Toda hierarchy *
}

\author{
L. Martínez Alonso ${ }^{1}$ and E. Medina ${ }^{2}$ \\ ${ }^{1}$ Departamento de Física Teórica II, Universidad Complutense \\ E28040 Madrid, Spain \\ 2 Departamento de Matemáticas, Universidad de Cádiz \\ E11510 Puerto Real, Cádiz, Spain
}

\begin{abstract}
Critical points of semiclassical expansions of solutions to the dispersionful Toda hierarchy are considered and a double scaling limit method of regularization is formulated. The analogues of the critical points characterized by the strong conditions in the Hermitian matrix model are analyzed and the property of doubling of equations is proved. A wide family of sets of critical points is introduced and the corresponding double scaling limit expansions are discussed.
\end{abstract}

Key words: Toda hierarchy. Double scaling limit. Hermitian matrix model. PACS number: 02.30.Ik.

*Partially supported by MEC project FIS2005-00319 and ESF programme MISGAM 


\section{Introduction}

In a recent work [1] we considered the large $N$ expansion of the Hermitian matrix model

$$
Z_{N}(\boldsymbol{t})=\int \mathrm{H} \exp \left(\frac{N}{g} \operatorname{tr}\left(\sum_{k \geq 1} t_{k} H^{k}\right)\right), \quad H=H^{\dagger},
$$

from the point of view of the dispersionful Toda hierarchy [2]

$$
\epsilon \frac{\partial \mathcal{L}}{\partial t_{j}}=\left[\left(\mathcal{L}^{j}\right)_{+}, \mathcal{L}\right], \quad \mathcal{L}=\Lambda+u+v \Lambda^{-1}, \quad \Lambda:=\exp \left(\epsilon \partial_{x}\right), \quad \epsilon:=g / N,
$$

where ()$_{+}$denotes the polynomial part in $\Lambda$. If the equilibrium measure in the large $N$ limit is supported on a single interval (one-cut case), then the partition function becomes a tau-function $\tau(x, \boldsymbol{t})$ of this hierarchy at $x=g$. The corresponding solution of (2) is characterized by two string equations which can be solved in terms of a semiclassical expansion

$$
u=\sum_{k \geq 0} \epsilon^{k} u^{(k)}(x, \boldsymbol{t}), \quad v=\sum_{k \geq 0} \epsilon^{2 k} v^{(2 k)}(x, \boldsymbol{t}) .
$$

The coefficients $\left(u^{(k)}, v^{(2 k)}\right)$ can be expressed as rational functions of the leading terms $\left(u^{(0)}, v^{(0)}\right)$ and their derivatives with respect to $x$.

A method to determine the expansions (3) was provided in [1. It uses two functions

$$
\mathbb{R}:=\sum_{k \geq 0} \frac{R_{k}(u, v)}{z^{k}}, \quad \mathbb{T}:=\sum_{k \geq 0} \frac{T_{k}(u, v)}{z^{k}}, \quad R_{0}=T_{0}=1,
$$

which are related to the resolvent $\mathcal{R}:=(z-\mathcal{L})^{-1}$ of the Lax operator $\mathcal{L}$ through the equation

$$
\left(z-\frac{2 \mathbb{R}}{(1+\mathbb{T})} \Lambda\right) \mathcal{R}_{+}=\mathbb{R}
$$

so that they generate the trace densities $\operatorname{Res}\left(\mathcal{R} \Lambda^{-j}\right)$ for $j \geq 0$, where $\operatorname{Res}\left(\sum c_{k} \Lambda^{k}\right):=c_{0}$. These functions are determined by the equations

$$
\left\{\begin{array}{l}
\mathbb{T}+\mathbb{T}_{[-1]}+\frac{2}{z}(u-z) \mathbb{R}=0 \\
\mathbb{T}^{2}-\frac{4}{z^{2}} v_{[1]} \mathbb{R} \mathbb{R}_{[1]}=1
\end{array}\right.
$$

where we are denoting

$$
F_{[r]}(x):=F(x+r \epsilon) .
$$

As it was shown in [1] the string equations for $(u, v)$ reduce to the system

$$
\left\{\begin{array}{l}
\oint_{\gamma} \frac{d z}{2 \pi i z} V_{z}(z, \boldsymbol{t}) \mathbb{R}(z, u, v)=0 \\
\oint_{\gamma} \frac{d z}{2 \pi i} V_{z}(z, \boldsymbol{t}) \mathbb{T}_{[-1]}(z, u, v)=-2 x
\end{array}\right.
$$


where $V(z, \mathbf{t}):=\sum_{j=1}^{\infty} t_{j} z^{j}$, and $\gamma$ is a large positively oriented closed path. For $\epsilon=0$ the system (5) reduces to a pair of hodograph type equations

$$
f_{v}\left(\boldsymbol{t}, u^{(0)}, v^{(0)}\right)=0, \quad f_{u}\left(\boldsymbol{t}, u^{(0)}, v^{(0)}\right)=2 x,
$$

where

$$
f(\boldsymbol{t}, u, v):=\frac{1}{2 \pi i} \oint_{\gamma} d z V_{z}(z, \boldsymbol{t})\left((z-u)^{2}-4 v\right)^{\frac{1}{2}}=-2 v t_{1}-4 u v t_{2}+\ldots
$$

These equations determine $\left(u^{(0)}, v^{(0)}\right)$ and represent the planar limit contribution to the partition function of the Hermitian model [6]. However, near critical points of (6) the functions $\left(u^{(0)}, v^{(0)}\right)$ are multi-valued and have singular $x$-derivatives (gradient catastrophe), so that the semiclassical expansion (3) breaks down. In terms of the Hermitian matrix model this situation corresponds to the critical points of the large $N$ expansion of $Z_{N}(\boldsymbol{t})$ and, as it is well-known [3]-[12], a double scaling limit method of regularization is available. This method leads to an important nonperturbative approach to two-dimensional quantum gravity.

Recent research [13]-[16] shows that the double scaling limit expansions are also relevant in the asymptotic analysis of solutions of dispersionful integrable systems. They supply good approximations in the transition regions from the semiclassical to the oscillatory regimes after the time of gradient catastrophe. These applications motivate the interest in reconsidering the double scaling limit method from the point of view of the theory of string equations for integrable systems of dispersionful type. The formulation of the double scaling limit within the theory of the dispersionful Toda hierarchy was already addressed in [1, but only the case corresponding to $u=0$ and even potentials $V(-z)=V(z)$ was analyzed. The purpose of the present paper is to analyze the general case that exhibits a much richer structure of critical behaviours (see [10]-[12] for its relevance in the Hermitian matrix model of random surfaces and quantum gravity).

According to the strategy of the double scaling method we look at the systems (44)-(5) as singular perturbation problems in the small parameter $\epsilon$ and consider the semiclassical expansions near critical points as outer expansions of the solutions of (4)-(5). Consequently the problem consists in characterizing appropriate inner expansions

$$
x=x_{c}+\bar{\epsilon}^{2 n} \bar{x}, \quad u=u_{c}+\sum_{j \geq 2} \bar{\epsilon}^{j} \bar{u}^{(j)}, \quad v=v_{c}+\sum_{j \geq 2} \bar{\epsilon}^{j} \bar{v}^{(j)}, \quad \epsilon=\bar{\epsilon}^{2 n+1}
$$

with coefficients depending on stretched variables $(\bar{x}, \overline{\boldsymbol{t}})$. This type of expansions were determined in the theory of random matrix models through several methods of analysis of discrete string equations for orthogonal polynomials in the large $N$ limit. The present work provides an alternative scheme based on the characterization of solutions

$$
\mathbb{R}=\sum_{j \geq 0} \bar{\epsilon}^{j} \bar{R}^{(j)}, \quad \mathbb{T}=\sum_{j \geq 0} \bar{\epsilon}^{j} \bar{T}^{(j)},
$$

of the resolvent trace equations (4) of the dispersionful Toda hierarchy. This scheme establishes the existence of the series (8) to all orders in $\bar{\epsilon}$ and leads to the characterization of the subleading coefficients $\left(\bar{u}^{(2)}, \bar{v}^{(2)}\right)$ in terms of ordinary differential equations involving the Gel'fand-Dikii polynomials of the resolvent trace expansion of the Schrödinger operator.

We analyze two different classes of critical points. Firstly, we consider the $\operatorname{sets} \mathcal{C}_{n}$ of critical points which underlie the Hermitian matrix model of quantum gravity [10]-[11] and we determine 
inner expansions (8) which satisfy the doubling property

$$
c_{ \pm} G_{n, \pm}\left(u_{ \pm}\right)=\bar{x}, \quad u_{ \pm}:=v^{(2)} \pm \sqrt{v_{c}} u^{(2)},
$$

with $G_{n, \pm}$ being the Gel'fand-Dikii polynomials. Secondly, we characterize a wide family of sets $\mathcal{C}_{l m n}$ of critical points where $l \geq 1, m=1, \ldots, l$ and $n \geq 2$, describe the branching behavior of $\left(u^{(0)}, v^{(0)}\right)$ near the critical points. Then we develop the double scaling limit method for the case $n=2$ with stretched variables

$$
x=x_{c}+\bar{\epsilon}^{4} \bar{x}, \quad t_{j}=t_{c, j}+\bar{\epsilon}^{4} \bar{t}_{j}, \quad j \geq 1, \quad \bar{\epsilon}=\epsilon^{1 / 5} .
$$

For $\mathcal{C}_{112}$ we find that the subleading coefficients satisfy

$$
\bar{v}^{(2)}= \pm \sqrt{v_{c}} \bar{u}^{(2)},
$$

with $\bar{v}^{(2)}$ being determined by the Painlevé I equation. For the other cases $\mathcal{C}_{l m 2}, l \geq 2$, we find again the doubling property. Indeed, the functions

$$
u_{ \pm}:=\bar{v}^{(2)} \pm \sqrt{v_{c}} \bar{u}^{(2)}
$$

turn out to be determined by a pair of decoupled Painlevé I equations.

The double scaling limit expansions at critical points of the types $\mathcal{C}_{l m n}$ can be applied to the asymptotic analysis of solutions of the dispersionful Toda hierarchy. As an illustrative example we end our work by applying our results to regularize some critical processes of ideal models of Hele-Shaw flows [18]-[20] based on the Toda hierarchy.

Our scheme can be formulated with other scaling ansätze for stretched variables like those of Galilean type used in [13]-[16]. Furthermore, it can be applied to the study of the double scaling limit of the normal matrix model which is related to another solution of the two-dimensional dispersionful Toda hierarchy [21]-23] determined by a pair of string equations. We also expect the methods of this paper to be applicable to dispersionful versions of the multi-component KP hierarchies which arise in random matrix theory and Dyson Brownian motions [26].

The layout of the paper is as follows: in section 2 we show how inner expansions of the generating functions $\mathbb{R}$ and $\mathbb{T}$ can be determined from the resolvent trace equations (44). In section 3 we characterize the double scaling limit expansions at critical points satisfying strong conditions. Finally, we devote section 4 to introduce families $\mathcal{C}_{l m n}$ of critical points and to develop the corresponding double scaling limit method.

\section{Inner expansions of the resolvent functions}

Let us consider the hodograph system (6)

$$
f_{v}=0, \quad f_{u}=2 x .
$$

Notice that the function $f$ defined in (7) satisfies

$$
f_{u u}-v f_{v v}=0 .
$$


The hodograph system determines a solution of the dispersionless Toda hierarchy provided that the implicit function theorem applies for obtaining $(u(x, \boldsymbol{t}), v(x, \boldsymbol{t}))$ from (9). For example (9) implies

$$
u_{t_{1}}=v_{x}, \quad v_{t_{1}}=v u_{x}
$$

so that

$$
v_{x x}-(\log v)_{t_{1} t_{1}}=0,
$$

which is the hyperbolic version of the long wave limit of the Toda lattice equation. It is also easy to see that the solutions of (9) verify

$$
u_{t_{2}}=2\left(u u_{t_{1}}+v_{t_{1}}\right), \quad v_{t_{2}}=2(v u)_{t_{1}},
$$

which is the dispersionless limit of the focusing nonlinear Schrödinger equation [16].

The set of critical points $\left(x_{c}, \boldsymbol{t}_{c}, u_{c}, v_{c}\right)$ of (9) is characterized by the system

$$
f_{v}=0, \quad f_{u}=2 x \quad f_{u v}^{2}-f_{u u} f_{v v}=0,
$$

Our main aim in this work is to characterize inner expansions for solutions $(u, v)$ of the system (41)-(5) near critical points of (9).

\subsection{Symmetric variables}

Let us start by analyzing the resolvent trace system (4). It is form invariant under the change of variables

$$
x=x_{c}+\bar{\epsilon}^{2 n} \bar{x}, \quad \epsilon=\bar{\epsilon}^{2 n+1}, \quad n \geq 2 .
$$

Indeed since $\epsilon \partial_{x}=\bar{\epsilon} \partial_{\bar{x}}$ we can rewrite (44) as

$$
\left\{\begin{array}{l}
\mathbb{T}+\mathbb{T}_{[-1]}+\frac{2}{z}(u-z) \mathbb{R}=0 \\
\mathbb{T}^{2}-\frac{4}{z^{2}} v_{[\overline{1}]} \mathbb{R}_{[\overline{1}]}=1
\end{array}\right.
$$

where we are denoting

$$
F_{[\bar{r}]}(\bar{x}):=F(\bar{x}+r \bar{\epsilon}) .
$$

We will prove that if $(u, v)$ are expansions of the form

$$
u=u_{c}+\sum_{j \geq 2} \bar{\epsilon}^{j} \bar{u}^{(j)}, \quad v=v_{c}+\sum_{j \geq 2} \bar{\epsilon}^{j} \bar{v}^{(j)}
$$

then the solution of (13) can be expressed as

$$
\mathbb{T}=\sum_{j \geq 0} \bar{\epsilon}^{j} \bar{T}^{(j)}, \quad \mathbb{R}=\sum_{j \geq 0} \bar{\epsilon}^{j} \bar{R}^{(j)}
$$

where the coefficients $\left(\bar{T}^{(j)}, \bar{R}^{(j)}\right)$ are differential polynomials in the coefficients of the expansions of $(u, v)$. 
An important property of the combined system (44)-(5) is its invariance under the transformation

$$
\begin{aligned}
& \widehat{u}(\bar{\epsilon}, \bar{x}):=u(-\bar{\epsilon}, \bar{x}+\bar{\epsilon}), \quad \widehat{v}(\bar{\epsilon}, \bar{x}):=v(-\bar{\epsilon}, \bar{x}), \\
& \widehat{\mathbb{T}}(\bar{\epsilon}, z, \bar{x}):=\mathbb{T}(-\bar{\epsilon}, z, \bar{x}+2 \bar{\epsilon}), \quad \widehat{\mathbb{R}}(\bar{\epsilon}, z, \bar{x}):=\mathbb{R}(-\bar{\epsilon}, z, \bar{x}+\bar{\epsilon})
\end{aligned}
$$

Thus it is convenient to introduce the symmetric variables

$$
\begin{gathered}
w(\bar{\epsilon}):=u\left(\bar{\epsilon}, \bar{x}-\frac{\bar{\epsilon}}{2}\right)=u_{[\overline{-1 / 2}]}(\bar{\epsilon}), \\
\mathbb{U}(\bar{\epsilon}):=\mathbb{T}(\bar{\epsilon}, z, \bar{x}-\bar{\epsilon})=\mathbb{T}_{[-1]}(\bar{\epsilon}) \quad \mathbb{V}(\bar{\epsilon}, z, \bar{x}):=\mathbb{R}\left(\bar{\epsilon}, z, \bar{x}-\frac{\bar{\epsilon}}{2}\right)=R_{[\overline{-1 / 2}]}(\bar{\epsilon}),
\end{gathered}
$$

which transform under (16) in the simple form

$$
\widehat{w}(\bar{\epsilon})=w(-\bar{\epsilon}), \quad \widehat{\mathbb{U}}(\bar{\epsilon}):=\mathbb{U}(-\bar{\epsilon}), \quad \widehat{\mathbb{V}}(\bar{\epsilon})=\mathbb{V}(-\bar{\epsilon}) .
$$

In terms of $(w, v, \mathbb{U}, \mathbb{V})$ the system (13) reads

$$
\left\{\begin{array}{l}
\mathbb{U}_{[\overline{1}]}+\mathbb{U}+\frac{2}{z}\left(w_{[\overline{1 / 2}]}-z\right) \mathbb{V}_{[\overline{1 / 2}]}=0, \\
\mathbb{U}^{2}-\frac{4}{z^{2}} v \mathbb{V}_{[\overline{-1 / 2}]} \mathbb{V}_{[\overline{1 / 2}]}=1 .
\end{array}\right.
$$

Moreover, from (20) one easily deduces the following linear equation for $\mathbb{U}$

$$
\begin{aligned}
\left(z-w_{[\overline{-1 / 2}]}\right) & \left(z-w_{[\overline{1 / 2}]}\right)\left(z-w_{[\overline{3 / 2}]}\right)\left(\mathbb{U}_{[\overline{1}]}-\mathbb{U}\right)=v_{[\overline{1}]}\left(z-w_{[\overline{-1 / 2}]}\right)\left(\mathbb{U}_{[\overline{2}]}+\mathbb{U}_{[\overline{1}]}\right) \\
& \left.-v\left(z-w_{[\overline{3 / 2}]}\right)\right)\left(\mathbb{U}_{[\overline{-1}]}+\mathbb{U}\right) .
\end{aligned}
$$

It can be expressed as

$$
\left(\sum_{k \geq 1} \bar{\epsilon}^{k} \mathcal{J}_{k}\right) \mathbb{U}=0
$$

where $\mathcal{J}_{k}$ are linear differential operators depending on the coefficients of the expansions of $(w, v)$ of the form

$$
\mathcal{J}_{k}=\left(z-u_{c}\right) r_{c} \mathcal{J}_{k, 0}+\left(z-u_{c}\right) \mathcal{J}_{k, 1}+r_{c} \mathcal{J}_{k, 2}+\mathcal{J}_{k, 3}
$$

where

$$
r_{c}=r_{c}(z):=\left(z-u_{c}\right)^{2}-4 v_{c},
$$

and $\mathcal{J}_{k, i}$ are $z$-independent. For example, the first few are

$$
\begin{aligned}
& \mathcal{J}_{1}=\left(z-u_{c}\right) r_{c} \partial_{\bar{x}}, \quad \mathcal{J}_{2}=\frac{1}{2}\left(z-u_{c}\right) r_{c} \partial_{\bar{x}}^{2} \\
& \mathcal{J}_{3}=\frac{z-u_{c}}{6} r_{c} \partial_{\bar{x}}^{3}-3 r_{c} u^{(2)} \partial_{\bar{x}}-\left(z-u_{c}\right)\left(v_{c} \partial_{\bar{x}}^{3}+4 v^{(2)} \partial_{\bar{x}}+2 v_{\bar{x}}^{(2)}\right)-4 v_{c}\left(2 u^{(2)} \partial_{\bar{x}}+u_{\bar{x}}^{(2)}\right) .
\end{aligned}
$$

We will now use both (20) and (21) to determine the expansions of $\mathbb{U}$ and $\mathbb{V}$. 
If we substitute in (20) the expansions

$$
\begin{aligned}
& w=u_{c}+\sum_{j \geq 2} \bar{\epsilon}^{j} \bar{w}^{(j)}, \quad v=v_{c}+\sum_{j \geq 2} \bar{\epsilon}^{j} \bar{v}^{(j)}, \\
& \mathbb{V}=\sum_{j \geq 0} \bar{\epsilon}^{j} \bar{V}^{(j)}, \quad \mathbb{U}=\sum_{j \geq 0} \bar{\epsilon}^{j} \bar{U}^{(j)},
\end{aligned}
$$

then by identifying the coefficients of the powers of $\bar{\epsilon}$ we find

$$
\frac{\bar{V}^{(0)}}{z}=\frac{1}{r_{c}^{1 / 2}}, \quad \bar{U}^{(0)}=\frac{z-u_{c}}{r_{c}^{1 / 2}}, \quad \frac{\bar{V}^{(1)}}{z}=\bar{U}^{(1)}=0,
$$

and the recurrence system $(j \geq 2)$

$$
\begin{aligned}
& \bar{U}^{(j)}-\left(z-u_{c}\right) \frac{\bar{V}^{(j)}}{z} \quad=-\frac{1}{2} \sum_{k+i=j, k \geq 1} \frac{1}{k !} \partial \frac{k}{\bar{x}} \bar{U}^{(i)}+\left(z-u_{c}\right) \sum_{k+i=j, k \geq 1} \frac{1}{2^{k}(k) !} \partial_{\bar{x}}^{\frac{k}{V^{(i)}}}- \\
& \sum_{\substack{i_{1}+i_{2}+k_{1}+k_{2}=j \\
i_{1} \geq 2}} \frac{1}{2^{k_{1}+k_{2}} k_{1} ! k_{2} !}\left(\partial_{\bar{x}}^{k_{1}} \bar{w}^{\left(i_{1}\right)}\right)\left(\partial_{\bar{x}}^{k_{2}} \frac{\bar{V}^{\left(i_{2}\right)}}{z}\right) \\
& \bar{U}^{(0)} \bar{U}^{(j)}-4 v_{c} \frac{\bar{V}^{(0)}}{z} \frac{\bar{V}^{(j)}}{z}=-\frac{1}{2} \sum_{i+k=j, 1 \leq i, k \leq j-1} \bar{U}^{(i)} \bar{U}^{(k)}+ \\
& \sum_{\substack{i_{1}+i_{2}+k_{1}+k_{2}=j \\
1 \leq i_{1}, i_{2} \leq j-1, k_{1}+k_{2} \text { even }}} \frac{(-1)^{k_{1}}}{2^{k_{1}+k_{2}-1} k_{1} ! k_{2} !} v_{c}\left(\partial_{\bar{x}}^{k_{1}} \frac{\bar{V}^{\left(i_{1}\right)}}{z}\right)\left(\partial_{\bar{x}}^{k_{2}} \frac{\bar{V}^{\left(i_{2}\right)}}{z}\right)+ \\
& \sum_{\substack{i_{1}+i_{2}+k_{1}+k_{2}+l=j \\
l \geq 2, k_{1}+k_{2} \text { even }}} \frac{(-1)^{k_{1}}}{2^{k_{1}+k_{2}} k_{1} ! k_{2} !} \bar{v}^{(l)}\left(\partial_{\bar{x}}^{k_{1}} \frac{\bar{V}^{\left(i_{1}\right)}}{z}\right)\left(\partial_{\bar{x}}^{k_{2}} \frac{\bar{V}^{\left(i_{2}\right)}}{z}\right)
\end{aligned}
$$

These equations are linear with respect to $\bar{U}^{(j)}$ and $\frac{\bar{V}^{(j)}}{z}$. Furthermore the determinant of their corresponding coefficients is $r_{c}^{1 / 2}$. 
Thus one obtains

$$
\begin{aligned}
& \bar{U}^{(2)}= \frac{1}{r_{c}^{\frac{3}{2}}}\left[4 v_{c} \bar{w}^{(2)}+2\left(z-u_{c}\right) \bar{v}^{(2)}\right], \quad \frac{\bar{V}^{(2)}}{z}=\frac{1}{r_{c}^{\frac{3}{2}}}\left[2 \bar{v}^{(2)}+\left(z-u_{c}\right) \bar{w}^{(2)}\right], \\
& \bar{U}^{(3)}= \frac{1}{r_{c}^{\frac{3}{2}}}\left[4 v_{c} \bar{w}^{(3)}+2\left(z-u_{c}\right) \bar{v}^{(3)}\right], \quad \frac{\bar{V}^{(3)}}{z}=\frac{1}{r_{c}^{\frac{3}{2}}}\left[2 \bar{v}^{(3)}+\left(z-u_{c}\right) \bar{w}^{(3)}\right], \\
& \bar{U}^{(4)}= \frac{1}{r_{c}^{\frac{3}{2}}}\left[4 v_{c} \bar{w}^{(4)}+\frac{v_{c}}{2} \bar{w}_{\overline{x x}}^{(2)}+4 \bar{v}^{(2)} \bar{w}^{(2)}+2\left(z-u_{c}\right) \bar{v}^{(4)}\right]+ \\
&+\frac{1}{r_{c}^{\frac{5}{2}}}\left[4 v_{c}^{2} \bar{w}_{\overline{x x}}^{(2)}+24 v_{c} \bar{v}^{(2)} \bar{w}^{(2)}+\left(z-u_{c}\right)\left(2 v_{c} \bar{v}_{\overline{x x}}^{(2)}+6 \bar{v}^{(2)^{2}}+6 v_{c} \bar{w}^{(2)^{2}}\right)\right], \\
& \frac{\bar{V}^{(4)}=}{z}\left[\frac{1}{r_{c}^{\frac{3}{2}}}\left[2 \bar{v}^{(4)}+\frac{1}{4} \bar{v}_{\overline{x x}}^{(2)}+\bar{w}^{(2)^{2}}+\left(z-u_{c}\right) \bar{w}^{(4)}\right]+\right. \\
& \quad \frac{1}{r_{c}^{\frac{5}{2}}}\left[2 v_{c} \bar{v}_{\overline{x x}}^{(2)}+6 v_{c} \bar{w}^{(2)^{2}}+6 \bar{v}^{(2)^{2}}+\left(z-u_{c}\right)\left(v_{c} \bar{w}_{\overline{x x}}^{(2)}+6 \bar{v}^{(2)} \bar{w}^{(2)}\right)\right],
\end{aligned}
$$

and by using induction one proves that the coefficients of the expansions of $\mathbb{U}$ and $\mathbb{V}$ are of the form

$$
\begin{aligned}
& \bar{U}^{(2 j)}=\sum_{l=1}^{j} \frac{\alpha_{l}^{(2 j)}\left(z-u_{c}\right)+\beta_{l}^{(2 j)}}{r_{c}^{l+\frac{1}{2}}}, \quad j \geq 3 ; \quad \bar{U}^{(2 j+1)}=\sum_{l=1}^{j} \frac{\alpha_{l}^{(2 j+1)}\left(z-u_{c}\right)+\beta_{l}^{(2 j+1)}}{r_{c}^{l+\frac{1}{2}}}, \quad j \geq 2, \\
& \frac{\bar{V}^{(2 j)}}{z}=\sum_{l=1}^{j} \frac{\gamma_{2 j l}\left(z-u_{c}\right)+\eta_{2 j l}}{r_{c}^{l+\frac{1}{2}}}, \quad j \geq 3 ; \quad \frac{\bar{V}^{(2 j+1)}}{z}=\sum_{l=1}^{j} \frac{\gamma_{l}^{(2 j+1)}\left(z-u_{c}\right)+\eta_{l}^{(2 j+1)}}{r_{c}^{l+\frac{1}{2}}}, \quad j \geq 2 .
\end{aligned}
$$

Moreover by substituting (24) in the linear equation (21) and taking into account (23) it can be proved by induction that the functions $\alpha_{l}^{(i)}, \beta_{l}^{(i)}, \gamma_{l}^{(i)}$ and $\eta_{l}^{(i)}$ are differential polynomials in $\left(\bar{v}^{(2)}, \ldots, \bar{v}^{(i-2 l+2)}, \bar{w}^{(2)}, \ldots, \bar{w}^{(i-2 l+2)}\right)$.

\section{$2.2 \quad$ Invariant solutions}

The solution $(\mathbb{U}, \mathbb{V})$ of the system $(20)$ is uniquely determined by $(w, v)$. Hence if we assume that $(w, v)$ are even functions of $\bar{\epsilon}$, then as a consequence of the invariance of (20) under the transformation (16) we deduce that $(\mathbb{U}, \mathbb{V})$ are even functions of $\bar{\epsilon}$ too. In this way we have

$$
w=u_{c}+\sum_{j \geq 1} \bar{\epsilon}^{2 j} \bar{w}^{(2 j)}, \quad v=v_{c}+\sum_{j \geq 1} \bar{\epsilon}^{2 j} \bar{v}^{(2 j)}, \quad \mathbb{U}=\sum_{j \geq 0} \bar{\epsilon}^{2 j} \bar{U}^{(2 j)}, \quad \mathbb{V}=\sum_{j \geq 0} \bar{\epsilon}^{2 j} \bar{V}^{(2 j)}
$$

These solutions will be henceforth called invariant solutions of the resolvent trace equations. 
As we have seen in the above subsection the coefficients $\bar{U}^{(2 j)}$ and $\bar{V}^{(2 j)}$ can be expanded in powers of $r_{c}$ with leading terms

$$
\bar{U}^{(2 j)}=\frac{\left(z-u_{c}\right) \alpha_{j}^{(2 j)}+\beta_{j}^{(2 j)}}{r_{c}^{j+\frac{1}{2}}}+\mathcal{O}\left(\frac{1}{r_{c}^{j-\frac{1}{2}}}\right), \quad \frac{\bar{V}^{(2 j)}}{z}=\frac{\left(z-u_{c}\right) \gamma_{j}^{(2 j)}+\eta_{j}^{(2 j)}}{r_{c}^{j+\frac{1}{2}}}+\mathcal{O}\left(\frac{1}{r_{c}^{j-\frac{1}{2}}}\right) .
$$

Furthermore, by identifying the coefficient of $\bar{\epsilon}^{2 j}$ in the first equation of the system (20) and by taking into account that $\left(z-u_{c}\right)^{2}=r_{c}+4 v_{c}$ it follows that

$$
\gamma_{j}^{(2 j)}=\frac{\beta_{j}^{(2 j)}}{4 v_{c}}, \quad \eta_{j}^{(2 j)}=\alpha_{j}^{(2 j)} .
$$

Now we prove the connection between these coefficients and the Gel'fand-Dikii differential polynomials of the KdV theory.

Theorem 1. The functions

$$
G_{j, \pm}:=\frac{\beta_{j}^{(2 j)}}{2 v_{c}} \pm \frac{\alpha_{j}^{(2 j)}}{\sqrt{v_{c}}}
$$

are the Gel'fand-Dikii differential polynomials in $u_{ \pm}:=v^{(2)} \pm \sqrt{v_{c}} u^{(2)}$, respectively.

Proof. By identifying the coefficient of $\bar{\epsilon}^{2 j+1}$ in (22) we get

$$
\sum_{k+2 l=2 j+1} \mathcal{J}_{k} \bar{U}^{(2 l)}=0
$$

From (23) and by taking into account that $\left(z-u_{c}\right)^{2}=r_{c}+4 v_{c}$ it is clear that only the terms $\mathcal{J}_{1} \bar{U}^{(2 j)}$ and $\mathcal{J}_{3} \bar{U}^{(2 j-2)}$ contribute to the coefficient of $\frac{1}{r_{c}^{j-\frac{1}{2}}}$ in (29). Thus we get the recursion relations

$$
\begin{aligned}
& \partial_{\bar{x}} \alpha_{j}^{(2 j)}=\left(v_{c} \partial_{\bar{x}}^{3}+4 \bar{v}^{(2)} \partial_{\bar{x}}+2 \bar{v}_{\bar{x}}^{(2)}\right) \alpha_{j-1}^{(2(j-1))}+\left(2 \bar{u}^{(2)} \partial_{\bar{x}}+\bar{u}_{\bar{x}}^{(2)}\right) \beta_{j-1}^{(2(j-1))}, \\
& \partial_{\bar{x}} \beta_{j}^{(2 j)}=\left(v_{c} \partial_{\bar{x}}^{3}+4 \bar{v}^{(2)} \partial_{\bar{x}}+2 \bar{v}_{\bar{x}}^{(2)}\right) \beta_{j-1}^{(2(j-1))}+4 v_{c}\left(2 \bar{u}^{(2)} \partial_{\bar{x}}+\bar{u}_{\bar{x}}^{(2)}\right) \alpha_{j-1}^{(2(j-1))},
\end{aligned}
$$

which lead at once to the well-known third-order differential equation for the Gelfand-Dikii differential polynomials

$$
\partial_{\bar{x}} G_{j, \pm}=\left(v_{c} \partial_{\bar{x}}^{3}+4 u_{ \pm} \partial_{\bar{x}}+2 u_{ \pm, \bar{x}}\right) G_{j-1, \pm}
$$

From (28) we have that $G_{1, \pm}= \pm \frac{2}{\sqrt{v_{c}}} u_{ \pm}$and by using (31) we find

$$
\begin{aligned}
G_{2, \pm}= \pm & \frac{2}{\sqrt{v_{c}}}\left(v_{c} u_{ \pm, \overline{x x}}+3 u_{ \pm}^{2}\right) \\
G_{3, \pm}= \pm & \frac{2}{\sqrt{v_{c}}}\left(v_{c}^{2} u_{ \pm, \overline{x x x x}}+10 v_{c} u_{ \pm, \overline{x x}} u_{ \pm}+5 v_{c} u_{ \pm, \bar{x}}^{2}+10 u_{ \pm}^{3}\right) \\
G_{4, \pm}= \pm & \frac{2}{\sqrt{v_{c}}}\left(v_{c}^{3} u_{ \pm, \overline{x x x x x x}}+14 v_{c}^{2} u_{ \pm} u_{ \pm, \overline{x x x x}}+28 v_{c}^{2} u_{ \pm, \overline{x x x}} u_{ \pm, \bar{x}}+21 v_{c}^{2} u_{ \pm, \overline{x x}}^{2}\right. \\
& \left.+70 v_{c} u_{ \pm}^{2} u_{ \pm, \overline{x x}}+70 v_{c} u_{ \pm} u_{ \pm, \bar{x}}^{2}+35 u_{ \pm}^{4}\right) .
\end{aligned}
$$




\section{Strong conditions for critical points and the dou- bling property}

In what follows the notation $F^{(c)}$ will represent the value of a function $F$ at a critical point $\left(x_{c}, \boldsymbol{t}_{c}, u_{c}, v_{c}\right)$ of (9). We will also suppose that $v_{c} \neq 0$. The following sets of critical points were considered in the applications of the Hermitian matrix model to quantum gravity [12]-[10].

Definition 1. Given $n \geq 2$ we denote by $\mathcal{C}_{n}$ the set of critical points of the hodograph system (9) which satisfy the (strong) conditions

$$
\left(\partial_{v}^{k} f\right)^{(c)}=\left(\partial_{u} \partial_{v}^{l} f\right)^{(c)}=0, \quad 1 \leq k \leq n, \quad 1 \leq l \leq n-1,
$$

and such that $\left(\left(\partial_{v}^{n+1} f\right)^{(c)},\left(\partial_{u} \partial_{v}^{n} f\right)^{(c)}\right) \neq(0,0)$.

Due to (10) it is clear that $\mathcal{C}_{n}$ is also determined by the condition that all derivatives $\left(\partial_{u}^{k} \partial_{v}^{l} f\right)^{(c)}$ with $(k, l) \neq(1,0)$ up to order $n$ vanish and such that at least one $n+1$-order derivative is different from zero.

An alternative characterization of $\mathcal{C}_{n}$ can be formulated in terms of the integrals

$$
I_{k}\left(\boldsymbol{t}_{c}, u_{c}, v_{c}\right):=\frac{1}{2 \pi i} \oint_{\gamma} d z \frac{V_{z}\left(z, \boldsymbol{t}_{c}\right)}{r_{c}^{k+1 / 2}}, \quad J_{k}\left(\boldsymbol{t}_{c}, u_{c}, v_{c}\right):=\frac{1}{2 \pi i} \oint_{\gamma} d z\left(z-u_{c}\right) \frac{V_{z}\left(z, \boldsymbol{t}_{c}\right)}{r_{c}^{k+1 / 2}} .
$$

Indeed, $I_{k}$ and $J_{k}$ are proportional to $\left(\partial_{v}^{k+1} f\right)^{(c)}$ and $\left(\partial_{u} \partial_{v}^{k} f\right)^{(c)}$ respectively. Hence it follows that

Lemma 1. $\left(x_{c}, \boldsymbol{t}_{c}, u_{c}, v_{c}\right) \in \mathcal{C}_{n}$ if and only if

$$
I_{0}=0, J_{0}=-2 x_{c}, \quad I_{k}=J_{k}=0, \quad \text { for } 1 \leq k \leq n-1, \quad\left(I_{n}, J_{n}\right) \neq(0,0) .
$$

Let us consider the system of string equations (5) at points $\left(x, \boldsymbol{t}_{c}, u, v\right)$ near a given critical point $\left(x_{c}, \boldsymbol{t}_{c}, u_{c}, v_{c}\right) \in \mathcal{C}_{n}$. In terms of symmetric variables it reads

$$
\left\{\begin{array}{l}
\oint_{\gamma} \frac{d z}{2 \pi i z} V_{z}\left(z, \boldsymbol{t}_{c}\right) \mathbb{V}(z)=0 \\
\oint_{\gamma} \frac{d z}{2 \pi i} V_{z}\left(z, \boldsymbol{t}_{c}\right) \mathbb{U}(z)=-2 x .
\end{array}\right.
$$

Then if we set

$$
x=x_{c}+\bar{\epsilon}^{2 n} \bar{x}, \quad \epsilon=\bar{\epsilon}^{2 n+1},
$$

and assume (25) we obtain a recursive method for determining the coefficients of $w$ and $v$. Indeed (35) is equivalent to

$$
\left\{\begin{array}{l}
\oint_{\gamma} \frac{d z}{2 \pi i z} V_{z}\left(z, \boldsymbol{t}_{c}\right) \bar{V}^{(2 j)}(z)=0 \\
\oint_{\gamma} \frac{d z}{2 \pi i} V_{z}\left(z, \boldsymbol{t}_{c}\right) \bar{U}^{(2 j)}(z)=-2 x_{c} \delta_{j 0}-2 \delta_{j n} \bar{x}
\end{array}\right.
$$


For $0 \leq j \leq n-1$ these equations are identically satisfied because of (34). For $j=n$ we get from (26) $-(27)$ that the equations (36) reduce to

$$
\left\{\begin{array}{l}
J_{n} \beta_{n}^{(2 n)}+4 v_{c} I_{n} \alpha_{n}^{(2 n)}=0 \\
I_{n} \beta_{n}^{(2 n)}+J_{n} \alpha_{n}^{(2 n)}=-2 \bar{x}
\end{array}\right.
$$

or, equivalently, in terms of the Gel'fand-Dikii polynomials (28) we obtain a pair of decoupled ordinary differential equations for $u_{ \pm}:=v^{(2)} \pm \sqrt{v_{c}} u^{(2)}$

$$
\left(J_{n} \pm 2 \sqrt{v_{c}} I_{n}\right) G_{n, \pm}\left(u_{ \pm}\right)=\mp \frac{2}{\sqrt{v_{c}}} \bar{x}
$$

Thus if the condition

$$
J_{n} \pm 2 \sqrt{v_{c}} I_{n} \neq 0
$$

is satisfied, we have the so-called doubling property arising in the one-cut case of the Hermitian matrix model [10]-[11].

The first few cases of (38) are

$$
\begin{aligned}
& \left(f_{u v v}^{(c)} \pm \sqrt{v_{c}} f_{v v v}^{(c)}\right)\left(v_{c} u_{ \pm, \overline{x x}}+3 u_{ \pm}^{2}\right)=12 \bar{x}, \quad n=2 \\
& \left(f_{u v v v}^{(c)} \pm \sqrt{v_{c}} f_{v v v v}^{(c)}\right)\left(v_{c}^{2} u_{ \pm, \overline{x x x x}}+10 v_{c} u_{ \pm, \overline{x x}} u_{ \pm}+5 v_{c} u_{ \pm, \bar{x}}^{2}+10 u_{ \pm}^{3}\right)=120 \bar{x}, \quad n=3 \\
& \left(f_{u v v v v}^{(c)} \pm \sqrt{v_{c}} f_{v v v v v}^{(c)}\right)\left(v_{c}^{3} u_{ \pm, \overline{x x x x x x}}+14 v_{c}^{2} u_{ \pm} u_{ \pm, \overline{x x x x}}+28 v_{c}^{2} u_{ \pm, \overline{x x x}} u_{ \pm, \bar{x}}+21 v_{c}^{2} u_{ \pm, \overline{x x}}^{2}\right. \\
& \left.\quad+70 v_{c} u_{ \pm}^{2} u_{ \pm, \overline{x x}}+70 v_{c} u_{ \pm} u_{ \pm, \bar{x}}^{2}+35 u_{ \pm}^{4}\right)=1680 \bar{x}, \quad n=4 .
\end{aligned}
$$

Finally for $j>n$ the system (36) yields the pair of equations

$$
\sum_{l=n}^{j}\left(J_{l} \gamma_{l}^{(2 j)}+I_{l} \eta_{l}^{(2 j)}\right)=0, \quad \sum_{l=n}^{j}\left(J_{l} \alpha_{l}^{(2 j)}+I_{l} \beta_{l}^{(2 j)}\right)=0
$$

which determine each pair $\left(w^{(2(j-n+1)}, v^{(2(j-n+1)}\right)$ recursively.

\section{Further critical points and regularized expansions}

Let us go back to the system (11) for critical points of the hodograph equations (91). It is equivalent to

$$
f_{v}=0, \quad f_{u}=2 x, \quad f_{u v}=\sigma \sqrt{v} f_{v v}, \quad \sigma= \pm 1 .
$$

Let us consider solutions of the hodograph equations near critical points and assume that

$$
f_{v}\left(\boldsymbol{t}_{c}, u, v_{c}\right) \not \equiv 0
$$

(similar results are obtained by interchanging the roles of $u$ and $v$ ). Then there exists an integer $l \geq 1$ verifying

$$
\left(\partial_{u}^{k} f_{v}\right)^{(c)}=0,(0 \leq k<l), \quad\left(\partial_{u}^{l} f_{v}\right)^{(c)} \neq 0 .
$$


As a consequence the first hodograph equation $f_{v}\left(\boldsymbol{t}_{c}, u, v\right)=0$ can be used to eliminate the variable $u$ as a function of $v$ near $\left(u_{c}, v_{c}\right)$. Indeed, from Weierstrass' preparation theorem it follows that near $\left(u_{c}, v_{c}\right)$ there exists a factorization

$$
f_{v}\left(\boldsymbol{t}_{c}, u, v\right)=\left(A_{0}\left(\boldsymbol{t}_{c}, v\right)+\ldots+A_{l-1}\left(\boldsymbol{t}_{c}, v\right) u^{l-1}+u^{l}\right) g\left(\boldsymbol{t}_{c}, u, v\right),
$$

where $A_{j}(0 \leq j<l)$ are analytic functions of $v$, and $g$ is an analytic function of $(u, v)$ which does not vanish near $\left(u_{c}, v_{c}\right)$. Hence the solutions of the hodograph equation $f_{v}\left(\boldsymbol{t}_{c}, u, v\right)=0$ near $\left(u_{c}, v_{c}\right)$ are given by the roots of the polynomial factor in (42)

$$
A_{0}\left(\boldsymbol{t}_{c}, v\right)+\ldots+A_{l-1}\left(\boldsymbol{t}_{c}, v\right) u^{l-1}+u^{l}=0,
$$

and consequently they are characterized by a Puiseux series

$$
u\left(\boldsymbol{t}_{c}, v\right)=u_{c}+\sum_{k \geq 1} a_{k}\left(\boldsymbol{t}_{c}\right)\left(v-v_{c}\right)^{\frac{k}{m}}
$$

for a certain integer $1 \leq m \leq l$. If we now introduce the function

$$
H\left(\boldsymbol{t}_{c}, w\right):=f_{u}\left(\boldsymbol{t}_{c}, u\left(\boldsymbol{t}_{c}, v_{c}+w^{m}\right), v_{c}+w^{m}\right), \quad\left(w:=\left(v-v_{c}\right)^{\frac{1}{m}}\right)
$$

then at $\boldsymbol{t}=\boldsymbol{t}_{c}$ the second hodograph equation in (9) reads $H\left(\boldsymbol{t}_{c}, w\right)=2 x$.

Furthermore it is easy to see that as a consequence of the system (40) we have

$$
H_{w}^{(c)}=f_{u u}^{(c)} u_{w}^{(c)}+f_{u v}^{(c)} v_{w}^{(c)}=\sigma \sqrt{v_{c}} f_{v v}^{(c)}\left(\sigma \sqrt{v_{c}} u_{w}^{(c)}+v_{w}^{(c)}\right) .
$$

On the other hand by differentiating the identity $f_{v}\left(\boldsymbol{t}_{c}, u\left(\boldsymbol{t}_{c}, v_{c}+w^{m}\right), v_{c}+w^{m}\right) \equiv 0$ we get

$$
f_{v u}^{(c)} u_{w}^{(c)}+f_{v v}^{(c)} v_{w}^{(c)}=f_{v v}^{(c)}\left(\sigma \sqrt{v_{c}} u_{w}^{(c)}+v_{w}^{(c)}\right)=0 .
$$

Hence, we deduce that

$$
H_{w}^{(c)}=0 .
$$

In this way if we assume that there exists an integer $n \geq 2$ such that

$$
\left(\partial_{w}^{k} H\right)^{(c)}=0,(1 \leq k<n), \quad\left(\partial_{w}^{n} H\right)^{(c)} \neq 0,
$$

then the hodograph equation $H\left(\boldsymbol{t}_{c}, w\right)=2 x$ determines $w$ as a function of $x$ with a branch point of order $n-1$ at $x=x_{c}$

Definition 2. We will denote by $\mathcal{C}_{l m n},(l \geq 1,1 \leq m \leq l, n \geq 2)$ the set of critical points of (9) characterized by (40)-(41) and such that

1. $u=u\left(\boldsymbol{t}_{c}, v\right)$ has branching order $m-1$ at $v=v_{c}$.

2. The corresponding function $H\left(\boldsymbol{t}_{c}, w\right)$ defined by (45) satisfies (47). 


\section{Example}

If we set $t_{n}=0$ for $n \neq 1,3$ then the system (40) reads

$$
t_{c 1}+3 t_{c 3}\left(u_{c}^{2}+2 v_{c}\right)=0, \quad 6 t_{c 3} u_{c} v_{c}+x_{c}=0, \quad u_{c}=\sigma \sqrt{v_{c}}, \quad \sigma= \pm 1 .
$$

Let us consider the critical points $\left(x_{c}, t_{c 1}, t_{c 3}, u_{c}, v_{c}\right)$ with $t_{c 3} \neq 0$. They are given by

$$
v_{c}=-\frac{t_{c 1}}{9 t_{c 3}}, \quad u_{c}=\sigma \sqrt{v_{c}},
$$

where $\left(x_{c}, t_{c 1}, t_{c 3}\right)$ are constrained by the equation

$$
3 x_{c}=2 \sigma t_{c 1}\left(-\frac{t_{c 1}}{9 t_{c 3}}\right)^{1 / 2} .
$$

As we are assuming that $v_{c} \neq 0$, we have that $t_{c 1} \neq 0$ and $u_{c} \neq 0$ so that (41) is satisfied by $l=1$. Moreover the hodograph equation

$$
t_{c 1}+3 t_{c 3}\left(u^{2}+2 v\right)=0
$$

leads to

$$
u\left(\boldsymbol{t}_{c}, w\right)=\left(-2\left(w-\frac{v_{c}}{2}\right)\right)^{1 / 2}, \quad w:=v-v_{c}, \quad v_{c}=-\frac{t_{c 1}}{9 t_{c 3}} \neq 0 .
$$

Furthermore $H\left(\boldsymbol{t}_{c}, w\right):=-12 t_{c 3} u\left(\boldsymbol{t}_{c}, w\right)\left(w+v_{c}\right)$ verifies $H_{w w}\left(\boldsymbol{t}_{c}, 0\right) \neq 0$. Therefore, it follows that $\left(x_{c}, t_{c 1}, t_{c 3}, u_{c}, v_{c}\right) \in \mathcal{C}_{112}$.

The following statements will be useful for the subsequent discussion. They are easily proved by differentiating (45) and the identity $f_{v}\left(\boldsymbol{t}, u(w), v_{c}+w^{m}\right)=0$.

Lemma 2. Given $\left(x_{c}, \boldsymbol{t}_{c}, u_{c}, v_{c}\right) \in \mathcal{C}_{l m n}$ with $v_{c} \neq 0$ then

- If $l=1$ then $f_{v v}^{(c)}$ does not vanish and

$$
H_{w w}^{(c)}=-\sigma v_{c}^{-1 / 2}\left(3 f_{v v}^{(c)}+4 v_{c} f_{v v v}^{(c)}-4 \sigma \sqrt{v_{c}} f_{u v v}^{(c)}\right) .
$$

- If $l \geq 2$ then $f_{u u}^{(c)}=f_{u v}^{(c)}=f_{v v}^{(c)}=0$ and

$$
H_{w w}^{(c)}= \begin{cases}v_{c} f_{u v v}^{(c)}\left(u_{w}^{(c)}\right)^{2}+2 v_{c} f_{v v v}^{(c)} u_{w}^{(c)}+f_{u v v}^{(c)}, & \text { for } \\ v_{c} f_{u v v}^{(c)}\left(u_{w}^{(c)}\right)^{2}, & \text { for } \quad m \geq 2,\end{cases}
$$

where $u_{w}^{(c)}$ satisfies

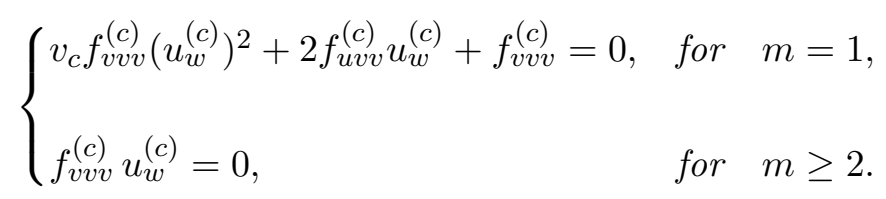

As a consequence we deduce the following conditions for critical points with $n=2$ 
Proposition 1. Given $\left(x_{c}, \boldsymbol{t}_{c}, u_{c}, v_{c}\right) \in \mathcal{C}_{l m 2}$ with $v_{c} \neq 0$, then it follows that

1. If $\left(x_{c}, \boldsymbol{t}_{c}, u_{c}, v_{c}\right) \in \mathcal{C}_{112}$, then

$$
3 \sigma f_{v v}^{(c)}+4 \sigma v_{c} f_{v v v}^{(c)}-4 \sqrt{v_{c}} f_{u v v}^{(c)} \neq 0 .
$$

2. If $\left(x_{c}, \boldsymbol{t}_{c}, u_{c}, v_{c}\right) \in \mathcal{C}_{l 12}$ with $l \geq 2$ then

$$
f_{u v v}^{(c)^{2}}-v_{c} f_{v v v}^{(c)^{2}} \neq 0
$$

3. If $\left(x_{c}, \boldsymbol{t}_{c}, u_{c}, v_{c}\right) \in \mathcal{C}_{l m 2}$ with $l, m \geq 2$ then

$$
f_{u v v}^{(c)} \neq 0, \quad \text { and } \quad f_{v v v}^{(c)}=0 .
$$

Proof. For $l=1$ we have that $m=1$ so that (53) is a consequence of (50). For $l \geq 2$ and $m=1$, by substituting the solution of (52) into (51) we obtain the condition (54).

Finally suppose that $l \geq 2, m \geq 2$. Then according to (52) and (51) we have that $u_{w}^{(c)} f_{v v v}^{(c)}=0$, and $H_{w w}^{(c)}=v_{c}\left(u_{w}^{(c)}\right)^{2} f_{u v v}^{(c)}$. Therefore the conditions for $H_{w w}^{(c)} \neq 0$ are $f_{v v v}^{(c)}=0$ and $f_{u v v}^{(c)} \neq 0$ which proves (55).

Let us consider the system of string equations (5) at points $(x, \boldsymbol{t}, u, v)$ near a given critical point $\left(x_{c}, \boldsymbol{t}_{c}, u_{c}, v_{c}\right) \in \mathcal{C}_{l m n}$. In terms of symmetric variables it reads

$$
\left\{\begin{array}{l}
\oint_{\gamma} \frac{d z}{2 \pi i z} V_{z}(z, \boldsymbol{t}) \mathbb{V}(z)=0, \\
\oint_{\gamma} \frac{d z}{2 \pi i} V_{z}(z, \boldsymbol{t}) \mathbb{U}(z)=-2 x .
\end{array}\right.
$$

One may introduce stretched variables not only for $x$ but also for $\boldsymbol{t}$. The most symmetrical choice is

$$
x=x_{c}+\bar{\epsilon}^{2 n} \bar{x} ; \quad t_{j}=t_{c, j}+\bar{\epsilon}^{2 n} \bar{t}_{j}, \quad j \geq 1 ; \quad \epsilon=\bar{\epsilon}^{2 n+1} .
$$

Notice that $\epsilon \partial_{t_{j}}=\bar{\epsilon} \partial_{\bar{t}_{j}}$ so that (12), (57) and

$$
u=u_{c}+\sum_{j \geq 2} \bar{\epsilon}^{j} \bar{u}^{(j)}, \quad v=v_{c}+\sum_{j \geq 2} \bar{\epsilon}^{j} \bar{v}^{(j)}
$$

are consistent with the Lax equations (2).

We will next concentrate on the case $n=2$ and will provide a recursive method for determining expansions of the form (58) near critical points in $\mathcal{C}_{l m 2}$. Thus we set

$$
x=x_{c}+\bar{\epsilon}^{4} \bar{x}, \quad t_{j}=t_{c, j}+\bar{\epsilon}^{4} \bar{t}_{j}, \quad j \geq 1, \quad \bar{\epsilon}=\epsilon^{1 / 5} .
$$

From (56), equating the coefficients of order $\bar{\epsilon}^{j}$ one finds

- For $0 \leq j \leq 3$ :

$$
\oint_{\gamma} \frac{d z}{2 \pi i} V_{z}\left(z, \boldsymbol{t}_{c}\right) \frac{\bar{V}^{(j)}}{z}=0, \quad \oint_{\gamma} \frac{d z}{2 \pi i} V_{z}\left(z, \boldsymbol{t}_{c}\right) \bar{U}^{(j)}=-2 x_{c} \delta_{j 0}
$$


- For $j \geq 4$ :

$$
\left\{\begin{array}{l}
\oint_{\gamma} \frac{d z}{2 \pi i} V_{z}\left(z, \boldsymbol{t}_{c}\right) \frac{\bar{V}^{(j)}}{z}+\oint_{\gamma} \frac{d z}{2 \pi i} V_{z}(z, \overline{\boldsymbol{t}}) \frac{\bar{V}^{(j-4)}}{z}=0 \\
\oint_{\gamma} \frac{d z}{2 \pi i} V_{z}\left(z, \boldsymbol{t}_{c}\right) \bar{U}^{(j)}+\oint_{\gamma} \frac{d z}{2 \pi i} V_{z}(z, \overline{\boldsymbol{t}}) \bar{U}^{(j-4)}=-2 \bar{x} \delta_{j 4} .
\end{array}\right.
$$

Let us first analyze the system (59). For $j=0$ it reduces to the hodograph equations

$$
f_{v}^{(c)}=0, \quad f_{u}^{(c)}=2 x_{c}
$$

and for $j=1$ is trivially verified since $\bar{U}^{(1)} \equiv \bar{V}^{(1)} \equiv 0$. Furthermore, it is straightforward to see that for $j=2$ both equations in (59) reduce to

$$
\left(\sigma \sqrt{v_{c}} \bar{u}^{(2)}+\bar{v}^{(2)}\right) f_{v v}^{(c)}=0 .
$$

Hence to proceed further it is required to distinguish the two types of critical points corresponding to $\mathcal{C}_{112}$ and $\mathcal{C}_{l m 2}$ with $l \geq 2$.

\section{Case $\mathcal{C}_{112}$}

In this case $f_{v v}^{(c)} \neq 0$ and (61) implies

$$
\bar{u}^{(2)}=\bar{w}^{(2)}=-\frac{\sigma}{\sqrt{v_{c}}} \bar{v}^{(2)} .
$$

For $j=3$ both equations in (59) lead to

$$
\bar{w}^{(3)}=-\frac{\sigma}{\sqrt{v_{c}}} \bar{v}^{(3)} .
$$

By setting $j=4$ in (60) we find that

$$
\begin{aligned}
\bar{w}^{(4)} & =-\frac{\sigma}{\sqrt{v_{c}}}\left(\bar{v}^{(4)}+\frac{1}{8} \bar{v}_{\overline{x x}}^{(2)}+\frac{1}{2 v_{c}} \bar{v}^{(2)^{2}}\right)-\frac{\sigma f_{v}\left(\overline{\boldsymbol{t}}, u_{c}, v_{c}\right)}{\sqrt{v_{c}} f_{v v}^{(c)}} \\
& +\frac{1}{6 f_{v v}^{(c)}}\left[f_{u v v}^{(c)}-\sigma \sqrt{v_{c}} f_{v v v}^{(c)}\right]\left(\bar{v}_{\overline{x x}}^{(2)}+\frac{6}{v_{c}} \bar{v}^{(2)^{2}}\right) .
\end{aligned}
$$

Moreover $\bar{v}^{(2)}=-\sigma \sqrt{v_{c}} \bar{u}^{(2)}$ must satisfy the Painlevé I equation

$$
\Gamma^{(c)}\left(\bar{v}_{\overline{x x}}^{(2)}+\frac{6}{v_{c}} \bar{v}^{(2)^{2}}\right)=6\left(2 \bar{x}-f_{u}\left(\overline{\boldsymbol{t}}, u_{c}, v_{c}\right)+\sigma \sqrt{v_{c}} f_{v}\left(\overline{\boldsymbol{t}}, u_{c}, v_{c}\right)\right) .
$$

where

$$
\Gamma^{(c)}:=-\sigma \frac{\sqrt{v_{c}}}{2}\left(3 f_{v v}^{(c)}+4 v_{c} f_{v v v}^{(c)}-4 \sigma \sqrt{v_{c}} f_{u v v}^{(c)}\right) .
$$

Notice that since $\left(x_{c}, \boldsymbol{t}_{c}, u_{c}, v_{c}\right) \in \mathcal{C}_{112}$ then Proposition 1 implies $\Gamma^{(c)} \neq 0$. 
To proceed further one uses induction in the recurrence system for $\bar{U}^{(j)}, \bar{V}^{(j)}$ to prove that the only coefficients in the expansions of $\bar{U}^{(j)}, \bar{V}^{(j)}$ depending on $\bar{v}^{(j)}, \bar{v}^{(j-1)}, \bar{v}^{(j-2)}, \bar{w}^{(j)}, \bar{w}^{(j-1)}$ and $\bar{w}^{(j-2)}$ are those corresponding to $l=1$ or 2 . Moreover one finds

$$
\begin{aligned}
\alpha_{1}^{(j)} & =2 \bar{v}^{(j)}+A_{1}^{(j)} \\
\alpha_{2}^{(j)} & =2 v_{c} \bar{v}_{\overline{x x}}^{(j-2)}+12 \bar{v}^{(2)} \bar{v}^{(j-2)}+12 v_{c} \bar{w}^{(2)} \bar{w}^{(j-2)}+A_{2}^{(j)}, \\
\beta_{1}^{(j)} & =4 v_{c} \bar{w}^{(j)}+\frac{v_{c}}{2} \bar{w}_{\overline{x x}}^{(j-2)}+4 \bar{v}^{(2)} \bar{w}^{(j-2)}+4 \bar{v}^{(j-2)} \bar{w}^{(2)}+B_{1}^{(j)} \\
\beta_{2}^{(j)} & \left.=4 v_{c}^{2} \bar{w}_{\overline{x x}}^{(j-2)}+24 v_{c} \bar{v}^{(2)} \bar{w}^{(j-2)}+\bar{v}^{(j-2)} \bar{w}^{(2)}\right)+B_{2}^{(j)}, \\
\gamma_{1}^{(j)} & =\bar{w}^{(j)}+C_{1}^{(j)}, \\
\gamma_{2}^{(j)} & =v_{c} \bar{w}_{\overline{x x}}^{(j-2)}+6 \bar{v}^{(2)} \bar{w}^{(j-2)}+6 \bar{v}^{(j-2)} \bar{w}^{(2)}+C_{2}^{(j)}, \\
\eta_{1}^{(j)} & =2 \bar{v}^{(j)}+\frac{1}{4} \bar{v}_{\overline{x x}}^{(j-2)}+2 \bar{w}^{(2)} \bar{w}^{(j-2)}+D_{1}^{(j)}, \\
\eta_{2}^{(j)} & =2 v_{c} \bar{v}_{\overline{x x}}^{(j-2)}+12 v_{c} \bar{w}^{(2)} \bar{w}^{(j-2)}+12 \bar{v}^{(2)} \bar{v}^{(j-2)}+D_{2}^{(j)} .
\end{aligned}
$$

where $A_{i}^{(j)}, B_{i}^{(j)}, C_{i}^{(j)}$ and $D_{i}^{(j)}, j \geq 5, i=1,2$ are differential polynomials in $\bar{v}^{(2)}, \ldots, \bar{v}^{(j-3)}, \bar{w}^{(2)}, \ldots, \bar{w}^{(j-3)}$. Then, for $j \geq 5$ substituting (24) into the first equation in (60) yields expressions

$$
\bar{w}^{(j)}=-\frac{\sigma}{\sqrt{v_{c}}} \bar{v}^{(j)}+K_{j}\left(\overline{\boldsymbol{t}}, \bar{v}^{(2)}, \ldots, \bar{v}^{(j-2)}\right),
$$

where $K_{j}$ are differential polynomials in $\bar{v}^{(2)}, \ldots, \bar{v}^{(j-2)}$. Moreover, both equations in (60) imply that $\bar{v}^{(j-2)}$ must verify a second order linear differential equation of the form

$$
\Gamma^{(c)}\left(\bar{v}_{\overline{x x}}^{(j-2)}+\frac{12}{v_{c}} \bar{v}^{(2)} \bar{v}^{(j-2)}\right)=H_{j-2}\left(\overline{\boldsymbol{t}}, \bar{v}^{(2)}, \ldots, \bar{v}^{(j-3)}\right)
$$

where $H_{j-2}$ are differential polynomials in $\bar{v}^{(2)}, \ldots, \bar{v}^{(j-3)}$. In this way, we have a scheme for determining the coefficients $\bar{u}^{(j)}, \bar{w}^{(j)}$ in (14).

We notice that if $\left\{\bar{v}^{(l)}, l \geq 2\right\}$ is a solution of (62)- $(63)(j \geq 5)$, then as a consequence of the symmetry transformation (16)-(19) we have that $\left\{(-1)^{l} \bar{v}^{(l)}, l \geq 2\right\}$ is also a solution of these equations. Consequently, the differential polynomials $H_{2 l+1}, l \geq 1$, in the right hand side of (63) are odd polynomials in $\bar{v}^{(2 j+1)}, 1 \leq j \leq l-1$ and their derivatives (for example $H_{3}\left(\overline{\boldsymbol{t}}, \bar{v}^{(2)}\right)=0$ ). Hence we may set $\bar{v}^{(2 j+1)} \equiv 0$ for all $j \geq 1$. Analogously, since the equations (62)-(63) can be written in terms of $\left\{\bar{w}^{(l)}, l \geq 2\right\}$, we may set $\bar{w}^{(2 j+1)} \equiv 0$ for all $j \geq 1$. 


\section{Case $\mathcal{C}_{l m 2}, l \geq 2$}

In this case the system (59) is trivially satisfied. Moreover, for $j=4$ we get from (60) that

$$
\left\{\begin{array}{l}
\Delta^{(c)}\left(v_{c} \bar{v}_{\overline{x x}}^{(2)}+3 \bar{v}^{(2)^{2}}+3 v_{c} \bar{w}^{(2)^{2}}\right)=6\left(f_{u v v}^{(c)}\left(f_{u}\left(\overline{\boldsymbol{t}}, u_{c}, v_{c}\right)-2 \bar{x}\right)-v_{c} f_{v v v}^{(c)} f_{v}\left(\overline{\boldsymbol{t}}, u_{c}, v_{c}\right)\right), \\
\Delta^{(c)}\left(v_{c} \bar{w}_{\overline{x x}}^{(2)}+6 \bar{w}^{(2)} \bar{v}^{(2)}\right)=6\left(f_{u v v}^{(c)} f_{v}\left(\overline{\boldsymbol{t}}, u_{c}, v_{c}\right)-f_{v v v}^{(c)}\left(f_{u}\left(\overline{\boldsymbol{t}}, u_{c}, v_{c}\right)-2 \bar{x}\right)\right)
\end{array}\right.
$$

where we are denoting

$$
\Delta^{(c)}:=v_{c}\left(f_{v v v}^{(c)}\right)^{2}-\left(f_{u v v}^{(c)}\right)^{2}
$$

From Proposition 1 we have that $\Delta^{(c)} \neq 0$ for critical points in $\mathcal{C}_{l m 2}$ with $l \geq 2$.

In terms of the variables $u^{( \pm)}=\bar{v}^{(2)} \pm \sqrt{v_{c}} \bar{u}^{(2)}$, the system (64) decouples into the two Painlevé I equations

$$
\left(f_{u v v}^{(c)} \pm \sqrt{v_{c}} f_{v v v}^{(c)}\right)\left(v_{c} u_{ \pm, \overline{x x}}+3\left(u_{ \pm}\right)^{2}\right)=6\left(2 \bar{x}-f_{u}\left(\overline{\boldsymbol{t}}, u_{c}, v_{c}\right) \mp \sqrt{v_{c}} f_{v}\left(\overline{\boldsymbol{t}}, u_{c}, v_{c}\right)\right) .
$$

Thus the doubling property is satisfied in this case.

In general, for $j \geq 5$ (60) leads to a second order linear system for $\bar{w}^{(j-2)}, \bar{v}^{(j-2)}$ of the form:

$$
\begin{aligned}
& 2 v_{c}\left(\bar{v}_{\bar{x} x}^{(j-2)}+6 \bar{w}^{(2)} \bar{w}^{(j-2)}\right)+12 \bar{v}^{(2)} \bar{v}^{(j-2)}=M_{j-2}\left(\bar{t}, \bar{w}^{(2)}, \ldots, \bar{w}^{(j-3)}, \bar{v}^{(2)}, \ldots, \bar{v}^{(j-3)}\right), \\
& v_{c} \bar{w}_{\overline{x x}}^{(j-2)}+6 \bar{w}^{(2)} \bar{v}^{(j-2)}+6 \bar{v}^{(2)} \bar{w}^{(j-2)}=N_{j-2}\left(\bar{t}, \bar{w}^{(2)}, \ldots, \bar{w}^{(j-3)}, \bar{v}^{(2)}, \ldots, \bar{v}^{(j-3)}\right) .
\end{aligned}
$$

where $M_{j-2}$ and $N_{j-2}$ are differential polynomials in $\bar{w}^{(2)}, \ldots, \bar{w}^{(j-3)}$ and $\bar{v}^{(2)}, \ldots, \bar{v}^{(j-3)}$. Thus, we have a recursive procedure to construct the coefficients $\bar{w}^{(j)}, \bar{v}^{(j)}, j \geq 2$.

Again, due to the symmetry (16)-(19), it is clear that if $\left(\bar{w}^{(j)}, \bar{v}^{(j)}\right),(j \geq 2)$ is solution of (65) then $\left((-1)^{j} \bar{w}^{(j)},(-1)^{j} \bar{v}^{(j)}\right),(j \geq 2)$ is also a solution of (65). Hence for odd $j$ the differential polynomials $M_{j-2}, N_{j-2}$ are odd polynomials in $\bar{w}^{(2 l+1)}, \bar{v}^{(2 l+1)}, l \geq 1$ and their $\bar{x}$-derivatives. Therefore, we may set $\bar{w}^{(2 l+1)}=0, \bar{v}^{(2 l+1)}=0$ for all $l \geq 1$.

\subsection{Critical processes in ideal Hele-Shaw flows}

In view of the properties of asymptotic solutions of the KdV equation [13]-[15] and the NLS equation [16] it should be expected that the inner expansions provided by the double scaling method will be relevant when the solutions of the dispersionless or the dispersionful Toda hierarchies reach a point of gradient catastrophe. We next consider an application to an ideal model of Hele-Shaw flows supplied by the Toda hierarchy.

A Hele-Shaw cell is a narrow gap between two plates filled with two fluids: say oil surrounding one or several bubbles of air. In the set-up considered in [18] (see also [19]-20]) air is injected in two fixed points of a simply-connected air bubble making the bubble break into two emergent bubbles. Before the break-off the interface oil-air remains free of cusp-like singularities and develops a smooth neck. The reversed evolution describes the merging of two bubbles. The analysis of [18] concludes that before the merging, the local structure of a small part of the interface containing the tips of the bubbles is described by a curve $Y=Y(X)$ which falls into universal classes characterized by two even integers $(4 n, 2), n \geq 1$, and a finite number $2 n$ of deformation parameters $t_{k}$. Assuming 
symmetry of the curve with respect to the $X$-axis, the general solution for the curve in the $(4 n, 2)$ class is

$$
Y(z):=\left(\frac{\sum_{k=1}^{2 n}(k+1) t_{k+1} z^{k}}{\sqrt{(z-a)(z-b)}}\right)_{\oplus} \sqrt{(z-a)(z-b)}, \quad X=z .
$$

where $a$ and $b$ are the positions of the bubbles tips. Due to the physical assumptions of the problem, the expansion

$$
Y(z)=\sum_{k=1}^{2 n}(k+1) t_{k+1} z^{k}+\sum_{k=0}^{\infty} \frac{Y_{n}}{z^{n}}, \quad z \rightarrow \infty
$$

must satisfy the conditions $Y_{0}=t$ (physical time) and $Y_{1}=0$ (bubble merging condition) which determine the positions $a, b$ of the tips. However if $Y_{1} \neq 0$ the evolution process leads to a critical point in which cusp-like singularities appear.

As it was shown in [18 the positions of the bubbles tips are determined by the pair of hodograph equations

$$
\sum_{k=1}^{\infty} k t_{k} r_{k-1}(u, v)=0, \quad \sum_{k=1}^{\infty} k t_{k} r_{k}(u, v)+2 x=0,
$$

where $t_{k}=0$ for $k>2 n, Y_{1}=2 x$ and

$$
r:=\frac{z}{\sqrt{(z-u)^{2}-4 v}}=\sum_{k \geq 0} \frac{r_{k}(u, v)}{z^{k}}, \quad a:=u-2 \sqrt{v}, \quad b:=u+2 \sqrt{v} .
$$

These are precisely the hodograph equations (6) . Thus the double scaling limit method can be used to regularize the solutions of (66)-(68) at critical points in terms of inner expansions of solutions of (5).

As an example let us analyze the critical process of a merging of two bubbles studied in section VII of [18]. We set $t:=t_{1}, t_{2}=0, t_{n}=0, n>3$ and fix $t_{3}$ to a given constant value $c$. Thus the Hele-Shaw interface is locally characterized by the curve

$$
Y(X)=3 c(X+u) \sqrt{(X-u)^{2}-4 v},
$$

and (68) reduces to

$$
t+3 c\left(u^{2}+2 v\right)=0, \quad 6 c v u+x=0 .
$$

This is the hodograph system (48) and its solution is given by

$$
\begin{aligned}
v= & \frac{t^{2}}{2^{2 / 3} 9 c \sqrt[3]{9 \sqrt{81 c^{2} x^{4}+4 c x^{2} t^{3}}-81 c x^{2}-2 t^{3}}}-\frac{t}{18 c} \\
& +\frac{\sqrt[3]{9 \sqrt{81 c^{2} x^{4}+4 c t^{3} x^{2}}-81 c x^{2}-2 t^{3}}}{18 \sqrt[3]{2} c}, \quad u=-\frac{x}{6 c v} .
\end{aligned}
$$

As we have seen above the critical points $\left(x_{c}, t_{c}, t_{c 3}, u_{c}, v_{c}\right)$ with $t_{c} \neq 0$ are in $\mathcal{C}_{112}$ so that our scheme can be applied to provide a dispersive regularization of (72) near critical points. by

Setting $x=x_{c}$ in (72) one finds that the outer approximations for $u$ and $v$ near $t=t_{c}$ are given

$$
u \sim u_{\text {out }}(t):=u_{c}-\frac{1}{3} \sqrt{\frac{1}{c}\left(t_{c}-t\right)}, \quad v \sim v_{\text {out }}(t):=v_{c}+\frac{u_{c}}{3} \sqrt{\frac{1}{c}\left(t_{c}-t\right)}, \quad \text { as } \quad t \rightarrow t_{c}^{-}
$$


where $u_{c}=\sigma \sqrt{v_{c}}$. Since we are considering a critical point in $\mathcal{C}_{112}$ we introduce the stretched variables

$$
x=x_{c}+\bar{\epsilon}^{4} \bar{x}, \quad t=t_{c}+\bar{\epsilon}^{4} \bar{t}
$$

and consider the inner expansions for $u$ and $v$

$$
u \sim u_{i n}:=u_{c}+\bar{\epsilon}^{2} \bar{u}^{(2)}\left(\bar{x}-u_{c} \bar{t}\right), \quad v \sim v_{i n}(\bar{t}):=v_{c}+\bar{\epsilon}^{2} \bar{v}^{(2)}\left(\bar{x}-u_{c} \bar{t}\right), \quad \text { as } \quad \bar{t} \rightarrow 0,
$$

where $\bar{u}^{(2)}=-\bar{v}^{(2)} / u_{c}$ and $\bar{v}^{(2)}$ verifies the Painlevé I equation

$$
\bar{v}_{\overline{x x}}^{(2)}+\frac{6}{u_{c}^{2}}\left(\bar{v}^{(2)}\right)^{2}=\frac{2}{3 c u_{c}}\left(\bar{x}-u_{c} \bar{t}\right) .
$$

The inner approximation at $\bar{x}=0$ must match the outer approximation in an overlap interval which has both $t-t_{c}$ small and $\bar{t}$ large. Writing the outer approximations (73) in terms of the inner variable $\bar{t}$ we have

$$
u_{\text {out }}(t)=u_{c}-\bar{\epsilon}^{2} \frac{1}{3} \sqrt{-\frac{\bar{t}}{c}}, \quad v_{\text {out }}(t)=v_{c}+\bar{\epsilon}^{2} \frac{u_{c}}{3} \sqrt{-\frac{\bar{t}}{c}} .
$$

Hence, it is clear that matching requires a solution $\bar{v}^{(2)}\left(\bar{x}-u_{c} \bar{t}\right)$ of $(\underline{75})$ satisfying

$$
\bar{v}^{(2)}\left(-u_{c} \bar{t}\right) \sim \frac{u_{c}}{3} \sqrt{-\frac{\bar{t}}{c}}, \quad \text { as } \quad \bar{t} \rightarrow-\infty .
$$

Now, if we introduce the change of variables

$$
W=-\left(\frac{3 c}{2 u_{c}^{2}}\right)^{2 / 5} \bar{v}^{(2)}, \quad \xi=\left(\frac{2}{3 c u_{c}^{3}}\right)^{1 / 5}\left(\bar{x}-u_{c} \bar{t}\right),
$$

it follows that $W$ must satisfy the P-I equation

$$
W_{\xi \xi}=6 W^{2}-\xi
$$

and

$$
W \sim-\sqrt{\frac{\xi}{6}}, \quad \text { as } \quad \xi \rightarrow \infty,
$$

so that $W$ must be the tritronquée solution of the P-I equation [24]-25]. Using a numerical approximation of $W$ one finds [27] that the regularized evolution is as follows: the right bubble develops a cusp, then a new bubble appears at this cusp and it grows until it merges with the tip of the left bubble. Finally, the tip of the left bubble absorbs the new one and stays joined to the right bubble.

\section{Acknowledgements}

The authors wish to thank the Spanish Ministerio de Educación y Ciencia (research project FIS2005-00319) and the European Science Foundation (MISGAM programme) for their support. 


\section{References}

[1] L. Martínez Alonso and E. Medina, J. Phys. A: Math. Gen. 40, 14223 (2007)

[2] K. Takasaki and T. Takebe, Rev. Math. Phys. 7, 743 (1995)

[3] E. Brezin and V. Kazakov, Phys. Lett. B 236, 144 (1990).

[4] M. Douglas and S. Shenker, Nuc. Phys. B 335, 635 (1990).

[5] D. Gross and A. Migdal,Phys. Rev. Lett. 64, 127 (1990)

[6] P. Di Francesco, P. Ginsparg and Z. Zinn-Justin, Phys. Rept. 254,1 (1995)

[7] A. S. Fokas, A. R. Its and A. V. Kitaev, Comm. Math. Phys. 147 , 395 (1992)

[8] P. M. Bleher and A. R. Its , Ann. Math. 150 , 185 (1999)

[9] P. M. Bleher and B. Eynard, J. Phys. A: Math. Gen. 36, 2085 (2003)

[10] P. M. S. Petropoulos, Phys. Lett. B 247, 363 (1990)

[11] C. Bachas and P. M. S. Petropoulos, Phys. Lett. B 261, 402 (1991)

[12] T. Hollowood, L. Miramontes, A. Pasquinucci and C: Nappi, Nucl. Phys. B 373, 247 (1992)

[13] T. Grava and C. Klein, Numerical solution of the small dispersion limit of Korteweg de Vries and Whitham equations arXiv:math-ph/0511011.

[14] T. Grava and C. Klein, Numerical study of a multiscale expansion of KdV and Camassa-Holm equation arXiv:math-ph/0702038.

[15] T. Claeys and T. Grava, Universality of the break-up profile for the KdV equation in the small dispersion limit using the Riemann-Hilbert approach arXiv:0801.2326v1 [math-phys].

[16] B. Dubrovin, T. Grava and C. Klein, On universality of critical behaviour in the focusing nonlinear Schõdinger equation, elliptic umbilic catastrophe and the tritronquée solution to the Painlevé-I equation arXiv:0704.0501[math.AP]15 May 2007.

[17] N. A. Kudryashov and M. B. Soukharev, Phys. Lett. A 237, 206 (1998)

[18] S-Y. Lee, E. Bettelheim and P. Wiegmann, Physica D 219, 23(2006).

[19] R. Teodorescu, P. Wiegmann and A. Zabrodin, Phys. Rev. Lett. 95, 044502 (2005).

[20] E. Bettelheim, P. Wiegmann, O. Agam and A. Zabrodin, Phys. Rev. Lett. 95, 244504 (2005).

[21] M. Mineev-Weinstein, P. Wiegmann and A. Zabrodin, Phys. Rev. Lett. 84, 5106 (2000).

[22] P. W. Wiegmann and P. B. Zabrodin, Comm. Math. Phys. 213 , 523 (2000).

[23] I. Krichever, M. Mineev-Weinstein, P. Wiegmann and A. Zabrodin, Physica D 198, 1 (2004).

[24] P. Boutroux, Les transcendents de Painlevé et le asymptotique des equations différentielles du 2-ordre. Ann. Ecole Norm, 30, 265 (1913).

[25] N. Joshi and A. Kitaev, On Boutroux's tritronquée solutions of the first Painlevé equation. Stud. Appl. Math. 107, 253 (2001).

[26] M. Adler, P. van Moerbeke and P. Vanhaecke, Moment matrices and multi-component KP, with applications to random matrix theory arXiv:math-ph/06122064.

[27] L. Martínez Alonso y E. Medina, Regularization of Hele-Shaw flows, multiscaling expansions and the Painleve I equation arXiv:math-ph/0710.3731 